\title{
COMPACTAÇÃO DO SOLO CAUSADA POR TRATORES FLORESTAIS NA COLHEITA DE Pinus taeda L. NA REGIÃO SUDOESTE DO PARANÁ ${ }^{1}$
}

\author{
Denise Andréia Szymczak², Eleandro José Brun³, Dalvan José Reinert ${ }^{4}$, Taciana Frigotto ${ }^{5}$, Carlos Cesar \\ Mazzalira $^{6}$, Alessandro Dal'Col Lúcio ${ }^{7}$ e José Marafiga ${ }^{8}$
}

\begin{abstract}
RESUMO - A compactação vem-se tornando um dos principais impactos causados ao solo pelas atividades de colheita florestal mecanizada. O peso e movimentação das máquinas no momento do corte e extração da madeira, aliados à condição de umidade do solo imprópria para tal, são as principais causas da degradação estrutural do solo, verificada, principalmente, por alterações em suas propriedades físicas. Nesse sentido, este trabalho visou identificar a compactação causada ao solo devido às operações de colheita florestal de Pinus taeda L. realizada em três diferentes níveis de umidade do terreno. O estudo foi realizado em área de uma empresa florestal localizada no Paraná sobre Latossolo Vermelho. Os tratamentos foram compostos pela interação de três fatores, sendo eles: umidade (colheita em dia chuva, três e sete dias após a chuva), operações de colheita (com passagem de máquinas, sem passagem/eventual queda de árvores e estaleiro), e a ocasião de coleta (antes e depois da colheita). A biomassa residual da colheita florestal foi essencial para minimizar a compactação do solo causada pelo tráfego das máquinas que foi próxima da máxima. A gradação de umidade representada pela colheita em diferentes números de dias após uma chuva não influenciou, de maneira diferenciada, a compactação do solo. Entretanto, as operações de colheita impactaram o solo até a profundidade de $10 \mathrm{~cm}$, causando a compactação nas linhas de tráfego das máquinas, enquanto as demais operações não provocaram alterações na densidade, macroporosidade e resistência à penetração.
\end{abstract}

Palavras-chave: Solos florestais; Colheita de madeira; Qualidade física do solo.

\section{SOIL COMPACTION CAUSED BY FOREST TRACTORS IN THE PinUS taeda L. HARVESTING IN THE SOUTH-WESTERN REGION OF PARANÁ}

\begin{abstract}
Soil compaction has been becoming one of the main impacts caused by mechanized harvesting activities. The weight and movement of machines at the time of cutting and loading timber combined with the inappropriate moisture condition of the soil are the main causes of soil structural degradation, observed mainly by changes in physical properties. Thus, this work aimed to identify soil compaction caused due to forest harvesting of Pinus taeda L. performed on three different soil moistures. The study was conducted in the operational areas of a forestry company located in Paraná on red latosol. The treatments consisted of the interaction of three factors: moisture (rainy day harvesting and 3 and 7 days after rain), harvesting operations (machine traffic, no machine traffic/eventual timer landing and yard), and time of collection (before and after harvest). The residual biomass from forest harvesting was essential to minimize soil compaction caused by machinery traffic that was close to the maximum. The gradation of soil moisture represented by the different
\end{abstract}

\footnotetext{
${ }^{1}$ Recebido em 12.05.2013 aceito para publicação em 04.05.2014.

${ }^{2}$ Programa de Pós-Graduação em Engenharia Florestal, Universidade Federal de Santa Maria, UFSM, Brasil. E-mail: <deniseszymczak@gmail.com>.

${ }^{3}$ Universidade Tecnológica Federal do Paraná, UTFPR, Brasil. E-mail: <eleandrobrun.utfpr@gmail.com>.

${ }^{4}$ Universidade Federal de Santa Maria, UFSM, Brasil. E-mail: <dalvan@ufsm.br>.

${ }^{5}$ Programa de Pós-Graduação em Engenharia Florestal da Universidade do Estado de Santa Catarina. E-mail: <tacianafrigotto@gmail.com>.

${ }^{6}$ Graduando em Engenharia Florestal, Universidade Tecnológica Federal do Paraná, UTFPR, Brasil. E-mail: <carlosmezzalira89@hotmail.com>.

${ }^{7}$ Universidade Federal de Santa Maria, UFSM, Brasil. E-mail: <adlucio@ufsm.br>.

${ }^{8}$ Araupel S/A. E-mail: <marafiga@araupel.com.br>.
} 
days of harvest after a rain did not influence differently the soil compaction. However, harvesting operations impacted soil to a depth of $10 \mathrm{~cm}$ causing compaction on the traffic lines of the machines, while the others operations did not cause changes in density, macroporosity and resistance to penetration.

Keywords: Forest soil; Timber harvesting; Soil physical quality.

\section{INTRODUÇÃO}

A modernização do setor de colheita florestal foi alavancada pela expansão da área de florestas plantadas, de maneira que esta se tornasse mais eficiente e econômica. Esse avanço tecnológico, iniciado na década de 1990, foi possível pela abertura do país às importações, permitindo que as empresas nacionais importassem tecnologia estrangeira. Atualmente, têm-se máquinas e equipamentos altamente produtivos e multifuncionais, porém cada vez mais pesados.

O intenso tráfego dessas máquinas dentro do povoamento, no momento dos desbastes e principalmente no corte raso, vem causando problemas, sobretudo, de ordem ambiental, em sua maioria relacionados à compactação do solo em áreas sob sistema de colheita mecanizada (SEIXAS et al., 1998; OLIVEIRA JUNIOR, 1998; DIAS JUNIOR, 1999; SAMPIETRO, 2011).

A compactação verificada no solo pode ser definida como incremento na densidade resultante da aplicação de cargas ou pressão. Esse aumento de densidade é influenciado pelo esforço/pressão de compactação e pela umidade do solo. A força requerida para compactar um solo decresce exponencialmente com a sua umidade (BAVER; GARDNER, 1973). Para Hillel (1998), o solo, quando sujeito a uma pressão, tende a se comprimir, isto é, a elevar sua densidade. O principal mecanismo de compressão do solo é a redução da sua porosidade, através da expulsão parcial de ar e, ou, água, a partir do solo comprimido.

A compactação do solo, dependendo do grau com que ocorre, pode reduzir a produtividade do sítio e aumentar os níveis de erosão. Lockaby e Vidrine (1984 apud CAMARGO, 1999) constataram que a compactação do solo próxima à superfície causou reduções no crescimento em altura de uma floresta de Pinus taeda, variando entre $40 \%$ e $60 \%$. Além dessas reduções de crescimento, alterações estruturais na morfologia da raiz de Araucaria angustifolia, com raízes superficiais, laterais finas e comprimento da raiz principal reduzido, foram relatadas por Mósea e Dillenburg (2004).
Diversos são os fatores que influenciam diretamente na compactação do solo, destacando-se o seu tipo e textura, o teor de umidade, o teor de matéria orgânica, a presença de resíduos florestais sobre a superfície, o tipo de máquinas e rodados e o número de passadas da máquina, entre outros (WARKOTSCH et al., 1994). Consequentemente, torna-se essencial a realização de estudos que auxiliem na compressão da interação desses fatores e, a partir disso, indicar aos gestores florestais a condição de colheita que menos impacta o solo e o ambiente como um todo.

O objetivo deste trabalho foi verificar a ocorrência da compactação do solo e quais os níveis que esse processo atinge após distintas operações de colheita de Pinus taeda L. realizadas em diferentes graus de umidade sobre um Latossolo Vermelho Distroférrico típico.

\section{MATERIAL E MÉTODOS}

\section{1. Área de estudo}

O trabalho foi realizado nas áreas operacionais de colheita florestal, em povoamentos de Pinus taeda L., pertencentes a uma empresa Araupel S/A, localizada no Estado do Paraná. O clima da região de estudo é classificado, de acordo com Köppen, como subtropical do tipo Cfa, com temperatura média anual de $20^{\circ} \mathrm{C}$, precipitação média anual entre 1.800 e $2.000 \mathrm{~mm}$, altitude de $530 \mathrm{~m}$, latitude de $25^{\circ} 27^{\prime} 22^{\prime \prime}$ 'S e longitude de $52^{\circ} 51^{\prime} 55^{\prime \prime} \mathrm{O}$ O (CAVIGLIONE et al., 2000).

O plantio de Pinus taeda L. ocorreu no ano 1990, no espaçamento $3 \mathrm{~m} \times 3 \mathrm{~m}$, passando por desramas em 1994 e 1997 e, em 2004, por um desbaste misto sistemático na quinta linha e seletivo nas demais. $\mathrm{O}$ povoamento, de segunda rotação, tinha 22 anos de idade na ocasião da colheita da madeira, com 382 árvores por hectare, volume médio de $450 \mathrm{~m}^{3} \mathrm{ha}^{-1}$, sendo o peso médio individual de cada árvore verde, acima do solo, de $0,95 \mathrm{t}$.

O solo da área foi classificado como Latossolo Vermelho Distroférrico típico de textura muito argilosa, com 667,91; 274,86; e 57,23 $\mathrm{g} \mathrm{kg}^{-1}$ de argila, silte e 
areia, respectivamente, e teor de carbono orgânico de $15,67 \mathrm{~g} \mathrm{~kg}^{-1}$ e $14,5 \mathrm{~g} \mathrm{~kg}^{-1}$, nas camadas de 0 a 5 e 5 a $10 \mathrm{~cm}$ de profundidade, respectivamente.

\subsection{Sistema de colheita de madeira}

O sistema de colheita da madeira utilizado pela empresa foi o de Toras Curtas (Cut to Length). A derrubada era realizada por um trator florestal harvester marca Caterpillar e modelo C9 ACERT, com peso operacional de 28 toneladas, com rodados de esteiras. A extração era realizada por trator florestal forwarder, marca Caterpillar e Modelo CAT 3056E DITAAC, com 23 toneladas, seu próprio peso somado à carga de madeira de cinco toneladas e rodados de pneus recobertos com semiesteiras na parte traseira.

\subsection{Coleta de dados}

Os tratamentos do estudo foram o resultado da combinação de três fatores: a) umidade: composto por colheita em dia de chuva (44,76\% de Ug), três dias após a chuva (42,18\% de Ug) e sete dias após a chuva (39,97\% de Ug); b) operações de colheita: composto por parcelas com uma passagem de harvester e três do forwarder carregado (CP), sem passagem de máquinas e com eventual queda da árvore no momento do corte (SP) e área de estaleiro das toras (E); e c) a ocasião de coleta das amostras: antes e depois da colheita florestal. A realização de uma coleta antes da colheita florestal foi necessária para representar a condição inicial do solo, tendo sido realizada na mesma estrutura amostral descrita, no entanto numa única condição de umidade e sem influência das operações de colheita.

Em cada tratamento, foram realizadas cinco repetições, representadas por parcelas de 20 m x 6 m. Em cada parcela, foram abertas duas trincheiras (40 $\mathrm{cm} \mathrm{x} 40 \mathrm{~cm}$ x $40 \mathrm{~cm}$ ), para a retirada de amostras de solo com estrutura preservada, nas profundidades de 0 a $5 \mathrm{~cm}, 5$ a $10 \mathrm{~cm}, 10$ a $20 \mathrm{~cm}$ e 20 a $30 \mathrm{~cm}$. O início da colheita ocorreu após uma chuva de aproximadamente $130 \mathrm{~mm}$. A umidade na capacidade de campo do solo, medida em laboratório, ficou próxima de 48\%.

Para determinação da densidade do solo (Ds) e da macroporosidade (Ma), foram coletadas amostras de solo com estrutura preservada com anéis volumétricos de 69,6 cm³ , em média; para determinação da umidade gravimétrica (Ug), utilizaram-se amostras com estrutura alterada, obtidas conforme a metodologia proposta pela EMBRAPA (1997).
A resistência do solo à penetração (RP) foi medida com penetrógrafo eletrônico digital, marca Falker ${ }^{\circledR}$ e modelo PLG1020, com profundidade máxima de penetração de $60 \mathrm{~cm}$, leituras de cm em cm, resolução configurável e diâmetro do cone de 12,83 mm. Utilizou-se como resultado a leitura média das camadas de 0 a 5,5 a 10, 10 a 20 e 20 a $30 \mathrm{~cm}$. Antes da colheita, a amostragem nas parcelas CP, SP e E foi aleatória. Depois da colheita, nas parcelas CP a medição da $\mathrm{RP}$ foi direcionada para o centro do rastro do rodado, e nas parcelas SP a amostragem foi aleatória. No estaleiro, a RP foi medida no centro da pilha de madeira. Para comparação da RP entre as três áreas de umidades diferentes, os dados foram ajustados por uma equação não linear proposta por Busscher (1990): RP = a x Ds ${ }^{\mathrm{b}} \times \mathrm{Uv}^{\mathrm{c}}$, em que RP = resistência à penetração; Ds = densidade do solo; Uv = umidade volumétrica do solo; e a, b e c = parâmetros empíricos da equação. O ajuste dos dados foi realizado no programa computacional SAS, obtendo-se um $\mathrm{R}^{2}=0,91$. A umidade volumétrica utilizada no ajuste foi de $0,418 \mathrm{~m}^{3} \cdot \mathrm{m}^{-3}$, que correspondeu à média das umidades nas áreas amostradas, e situava-se próxima à capacidade de campo.

Para quantificação da serapilheira e da biomassa residual, foi utilizada uma moldura de ferro quadrada com lado igual a $50 \mathrm{~cm}$, com as coletas realizadas de forma aleatória em cada parcela amostral, num total de 120 amostras antes e 120 depois da colheita. A média de serapilheira + biomassa residual ficou em torno de 90 toneladas no interior do talhão (CP e SP) e de cinco toneladas na beira da estrada (E).

\subsection{Análises estatísticas}

O experimento foi analisado adotando-se o delineamento inteiramente casualizado com repetições, composto em trifatorial com parcelas subsubdivididas no tempo. As diferentes umidades do solo compuseram o primeiro fator (parcela principal - PP); operações de colheita, o segundo fator (parcela subdividida - PS); e ocasião de coleta, o terceiro fator (parcela subsubdividida no tempo - PSS). Foi verificada a normalidade na distribuição dos resíduos seguindo o teste de Kolmogorov-Smirnov e, posteriormente, utilizado o teste de Scott-Knott para comparação das médias. As análises foram realizadas no programa estatístico Assistat e Sisvar a 5\% de probabilidade de erro.

Revista Árvore, Viçosa-MG, v.38, n.4, p.641-648, 2014 


\section{RESULTADOS}

O efeito da colheita florestal mecanizada causou impacto, em termos de compactação, apenas nas camadas superficiais do solo (Tabela 1) e sem diferenciação entre as áreas com diferentes umidades do solo. Nas camadas de 0 a 5 e 5 a $10 \mathrm{~cm}$ de profundidade, antes da colheita florestal, a densidade do solo (Ds) no interior do talhão (CP e SP) era homogênea, diferindo da área que receberia as madeiras (E). Após a realização da colheita florestal, esse cenário se alterou nas parcelas CP. A eventual queda e arraste de árvores (SP) não alteraram a Ds, assim como o acúmulo de madeiras no estaleiro (E). A variação ocorrida na densidade do solo, de 1,02 para $1,18 \mathrm{Mg} \mathrm{m}^{-3}$ na camada de 0 a $5 \mathrm{~cm}(15,7 \%)$ e de 1,03 para $1,17 \mathrm{Mg} \mathrm{m}^{-3}$ na camada de 5 a $10 \mathrm{~cm}(13,6 \%)$, em CP, significou a ocorrência de compactação do solo.

Para a macroporosidade do solo (Tabela 2) nas profundidades de 0 a 5 e 5 a $10 \mathrm{~cm}$, as atividades de colheita florestal causaram compactação verificada nas parcelas CP, com redução aproximada de 60 e 50\%, respectivamente, da macroporosidade em relação à condição inicial do solo.

Nas parcelas SP (0 a $5 \mathrm{~cm}$ ) houve efeito inverso, com maior macroporosidade verificada no pós-colheita. Isso pode ser efeito do alto teor de carbono orgânico presente no solo, que eleva a porosidade, como também pode ter ocorrido o revolvimento do solo pelo arraste da copa pelo cabeçote do harvester no momento do corte das árvores, sendo, coincidentemente nesse ponto, efetuada a coleta da amostra de solo. No estaleiro (E), a macroporosidade não sofreu influência significativa do peso da madeira.

Nas parcelas submetidas ao tráfego das máquinas de colheita (CP) em todas as profundidades, após a realização das operações, a macroporosidade ficou próxima (0 a $5 \mathrm{~cm}$ ) ou abaixo (demais profundidades) do mínimo considerado adequado ao crescimento das plantas, de 0,10 $\mathrm{m}^{3} \mathrm{~m}^{-3}$ (BAVER, 1973; TORMENA et al., 1998). Assim como ocorreu com a densidade e

Tabela 1 - Valores médios da densidade do solo $\left(\mathrm{Mg} \mathrm{m}^{-3}\right)$ em um Latossolo Vermelho sob plantio de Pinus taeda L. Table 1 - Soil density average $\left(\mathrm{Mg} \mathrm{m}^{-3}\right)$ in a Red Latosol under planting of Pinus taeda L.

\begin{tabular}{|c|c|c|c|c|c|c|c|c|}
\hline \multirow[t]{3}{*}{ Fatores } & \multicolumn{8}{|c|}{ Profundidade $(\mathrm{cm})$} \\
\hline & \multicolumn{2}{|c|}{$0-5$} & \multicolumn{2}{|c|}{$5-10$} & \multicolumn{2}{|c|}{$10-20$} & \multicolumn{2}{|c|}{$20-30$} \\
\hline & Antes & Depois & Antes & Depois & Antes & Depois & Antes & Depois \\
\hline CP & $1,02 \mathrm{~B}^{*} \mathrm{~b}^{*}$ & $1,18 \mathrm{Aa}^{*}$ & $1,03 \mathrm{Bb}^{*}$ & $1,17 \mathrm{Aa}^{*}$ & 1,10 & 1,13 & 1,13 & 1,12 \\
\hline SP & $1,04 \mathrm{Ba}$ & $1,04 \mathrm{Ca}$ & $1,06 \mathrm{Ba}$ & $1,05 \mathrm{Ca}$ & 1,10 & 1,07 & 1,08 & 1,06 \\
\hline $\mathrm{E}$ & $1,11 \mathrm{Aa}$ & $1,11 \mathrm{Ba}$ & $1,13 \mathrm{Aa}$ & $1,12 \mathrm{Ba}$ & 1,14 & 1,14 & 1,12 & 1,16 \\
\hline CV SP (\%) & \multicolumn{2}{|c|}{3,29} & \multicolumn{2}{|c|}{4,60} & \multicolumn{2}{|c|}{5,84} & \multicolumn{2}{|c|}{4,59} \\
\hline CV SSP (\%) & \multicolumn{2}{|c|}{3,07} & \multicolumn{2}{|c|}{4,43} & \multicolumn{2}{|c|}{6,18} & \multicolumn{2}{|c|}{4,98} \\
\hline
\end{tabular}

* Médias não seguidas por mesma letra maiúscula na coluna e minúscula na linha dentro de cada profundidade são diferentes entre si, pelo teste de Scott-Knott a 5\% de probabilidade de erro. SP - Subparcela e SSP - Subsubparcela.

* Means not followed by the same uppercase and lowercase column on line within each depth are different, by Scott Knott test at 5\% of error probability. SP - subplot e SSP - subsubplot.

Tabela 2 - Valores médios da macroporosidade do solo $\left(\mathrm{m}^{3} \mathrm{~m}^{-3}\right)$ em um Latossolo Vermelho sob plantio de Pinus taeda L. Table 2 - Soil macroporosity average $\left(\mathrm{m}^{3} \mathrm{~m}^{-3}\right)$ in a Red Latosol under planting of Pinus taeda $L$.

\begin{tabular}{|c|c|c|c|c|c|c|c|c|}
\hline \multirow[t]{3}{*}{ Fatores } & & \multicolumn{7}{|c|}{ Profundidade $(\mathrm{cm})$} \\
\hline & \multicolumn{2}{|c|}{$0-5$} & \multicolumn{2}{|c|}{$5-10$} & \multicolumn{2}{|c|}{$10-20$} & \multicolumn{2}{|c|}{$20-30$} \\
\hline & Antes & Depois & Antes & Depois & Antes & $\overline{\text { Depois }}$ & Antes & $\overline{\text { Depois }}$ \\
\hline $\mathrm{CP}$ & $0,186 \mathrm{Aa}^{*}$ & $0,107 \mathrm{C}^{*} \mathrm{~b}$ & $0,160 \mathrm{Aa}^{*}$ & $0,074 \mathrm{Cb}^{*}$ & 0,140 & 0,089 & 0,106 & 0,097 \\
\hline SP & $0,163 \mathrm{Ab}^{*}$ & $0,208 \mathrm{Aa}$ & $0,144 \mathrm{Aa}$ & $0,152 \mathrm{Aa}$ & 0,117 & 0,119 & 0,133 & 0,122 \\
\hline $\mathrm{E}$ & $0,168 \mathrm{Aa}$ & $0,147 \mathrm{Ba}$ & $0,119 \mathrm{Ba}$ & $0,116 \mathrm{Ba}$ & 0,106 & 0,091 & 0,101 & 0,078 \\
\hline CV SP (\%) & \multicolumn{2}{|c|}{26,99} & \multicolumn{2}{|c|}{26,60} & \multicolumn{2}{|c|}{26,60} & \multicolumn{2}{|c|}{13,14} \\
\hline CV SSP (\%) & \multicolumn{2}{|c|}{12,62} & \multicolumn{2}{|c|}{33,96} & \multicolumn{2}{|c|}{27,84} & \multicolumn{2}{|c|}{9,72} \\
\hline
\end{tabular}

* Médias não seguidas por mesma letra maiúscula na coluna e minúscula na linha dentro de cada profundidade são diferentes entre si, pelo teste de Scott-Knott a 5\% de probabilidade de erro. SP - Subparcela e SSP - Subsubparcela.

* Means not followed by the same uppercase and lowercase column on line within each depth are different, by Scott Knott test at $5 \%$ of error probability. SP - subplot e SSP - subsubplot.

Revista Árvore, Viçosa-MG, v.38, n.4, p.641-648, 2014 
macroporosidade do solo, a RP também evidenciou que a compactação causada ao solo efetivamente se deu nos primeiros $10 \mathrm{~cm}$ (Tabela 3). A RP medida antes da colheita florestal foi maior nas parcelas E, em comparação com o interior do povoamento (CP e SP), mostrando que essa área é diferente das demais por ser beira de estrada e sofrer a interferência de outras operações, como a recuperação de estradas, abertura e limpeza de aceiros. Após a colheita, nas parcelas CP foi registrado um incremento de RP da ordem de $29,5 \%$ na camada de 0 a 5 e de 21,9\% na de 5 a $10 \mathrm{~cm}$; nas demais parcelas, não se constatou diferença significativa em relação à condição original.

Os menores valores (1,13 e 1,17 MPa) de RP estiveram associados às parcelas SP e indicaram que a queda das árvores no momento do corte e o arraste proporcionado pelo cabeçote do harvester não foram suficientes para alterar a estrutura do solo.

\section{DISCUSSÃO}

O desenvolvimento radicular das plantas não foi afetado pelo incremento de densidade nas primeiras camadas de solo, proporcionado pelas atividades de colheita, pois este não atingiu a densidade considerada crítica para solos argilosos, que varia de 1,30 a 1,40 $\mathrm{Mg} \mathrm{m}^{-3}$ (REICHERT et al., 2003). Compactações superficiais do solo causadas pela colheita florestal também foram verificadas por Sampietro e Lopes (2011), em estudo de colheita com feller-buncher e skidder, os quais também afirmaram que o incremento nas camadas mais profundas foi, em média, 73,4\% menor do que na camada superficial do solo. Tal fato indicou que essa camada é mais suscetível à compactação, especialmente pela presença de material orgânico incorporado ao solo, que o torna mais poroso e mais frágil quando recebe cargas externas provenientes das máquinas da colheita. Além disso, a pressão aplicada pelo tráfego sobre o solo pode se dissipar e não ser suficiente para impactar as propriedades físicas das camadas mais profundas, em nível considerável (CULLEN et al., 1991).

A camada de resíduos da colheita que permaneceu à frente do harverster no momento do corte e processamento e do forwarder durante a extração favoreceu a dissipação da pressão nas parcelas CP, evitando que a compactação chegasse a níveis críticos e se prolongasse nas camadas subsuperficiais do solo. Nessa mesma linha, Silva et al. (2007), avaliando a passagem de um forwarder sobre a galhada, galhada mais a casca, solo sem resíduo e um local sem tráfego da máquina, concluíram que os resíduos florestais minimizaram a compactação do solo. O solo sem resíduo foi mais suscetível à compactação, e a presença de galhada mais a casca foi a condição que ofereceu maior resistência à compactação, pois dissipou parte da pressão exercida pelas máquinas, evitando que a capacidade de suporte do solo fosse atingida. A densidade maior na beira da estrada provocada por movimentações de máquinas e madeira em ocasiões passadas e a quantidade e forma de alocação das madeiras no estaleiro colaboraram para que a pressão exercida sobre o solo não causasse alteração na estrutura do solo. O mesmo comportamento foi verificado nas parcelas SP.

A macroporosidade do solo foi afetada pela passagem das máquinas nas trilhas de colheita que, geralmente, ocorre nas primeiras passadas. Silva et al. (2006) também encontraram grandes decréscimos na macroporosidade, na ordem de 53\%, em um Latossolo Vermelho-Amarelo, sob elevadas pressões. Essa

Tabela 3 - Valores médios de RP (MPa) em um Latossolo Vermelho sob plantio de Pinus taeda L. Table 3 - RP average (MPa) in a Red Latosol under planting of Pinus taeda $L$.

\begin{tabular}{|c|c|c|c|c|c|c|c|c|}
\hline \multirow[t]{3}{*}{ Fatores } & \multicolumn{8}{|c|}{ Profundidade $(\mathrm{cm})$} \\
\hline & \multicolumn{2}{|c|}{$0-5$} & \multicolumn{2}{|c|}{$5-10$} & \multicolumn{2}{|c|}{$10-20$} & \multicolumn{2}{|c|}{$20-30$} \\
\hline & Antes & Depois & Antes & Depois & Antes & Depois & Antes & Depois \\
\hline CP & $1,12 \mathrm{~B}^{*} \mathrm{~b}^{*}$ & $1,45 \mathrm{~A} * \mathrm{a}$ & $1,14 \mathrm{~B} * \mathrm{~b} *$ & 1,39 Аа & 1,29 & 1,31 & 1,34 & 1,31 \\
\hline SP & 1,13 $\mathrm{Ba}$ & $1,17 \mathrm{Ca}$ & $1,19 \mathrm{Ba}$ & 1,19 Ва & 1,26 & 1,25 & 1,27 & 1,23 \\
\hline $\mathrm{E}$ & $1,27 \mathrm{Aa}$ & $1,35 \mathrm{Ba}$ & $1,31 \mathrm{Aa}$ & 1,32 Aa & 1,36 & 1,41 & 1,34 & 1,39 \\
\hline CV SP (\%) & \multicolumn{2}{|c|}{3,65} & \multicolumn{2}{|c|}{7,55} & \multicolumn{2}{|c|}{8,74} & \multicolumn{2}{|c|}{7,67} \\
\hline CV SSP (\%) & \multicolumn{2}{|c|}{1,95} & \multicolumn{2}{|c|}{3,59} & \multicolumn{2}{|c|}{6,58} & \multicolumn{2}{|c|}{15,2} \\
\hline
\end{tabular}

* Médias não seguidas por mesma letra maiúscula na coluna e minúscula na linha dentro de cada profundidade são diferentes entre si, pelo teste de Scott-Knott a 5\% de probabilidade de erro. SP - Subparcela e SSP - Subsubparcela.

* Means not followed by the same uppercase and lowercase column on line within each depth are different, by Scott Knott test at $5 \%$ of error probability. SP - subplot e SSP - subsubplot. 
tendência foi semelhante à observada por Marsili et al. (1998), que verificaram decréscimo da macroporosidade na camada superficial ( 0 a $10 \mathrm{~cm}$ ) depois de uma passada de dois tratores, cujo efeito foi intensificado por quatro passadas das máquinas.

Conforme Klein (2012), os macroporos são os responsáveis pelos poros de aeração, visto que se esvaziam de água mais facilmente. Alterações na estrutura do solo ocasionadas pela compactação reduzem a porosidade de aeração. O efeito negativo dessa redução sobre o desenvolvimento das plantas ocorre por deficiência na difusão de gases no sistema radicular das plantas (STEPNIEWSKI et al., 1994). Alguns desses efeitos, segundo Sojka (1992), são: fechamento dos estômatos, murchamento das plantas, necrose das raízes, obstrução do xilema à água e desbalanço nutricional e hormonal das plantas. No estudo de Seixas e Oliveira Junior (2001), com colheita de madeira sobre um Argissolo úmido em módulo de colheita harvester e forwarder, em diferentes declividades, também identificaram problemas na macroporosidade na classe de declive $<15 \%$, com valor médio abaixo do limite crítico, confirmando a necessidade de cuidados adicionais com o solo no momento da colheita.

Os maiores incrementos de RP ocorreram nas trilhas de passagem de máquinas, pois, segundo Fenner (2002), a utilização do módulo de colheita harvester e forwarder implica tráfego concentrado nos ramais de extração de madeira. Outro módulo de colheita como fellerbuncher e skidder implica que, praticamente, toda a área será trafegada, pelo menos uma vez. Seixas et al. (2003) realizaram a instalação de aparelho GPS em um forwarder, concluindo que a movimentação do trator, na extração de madeira de povoamento de eucalipto em corte raso, foi de maneira concentrada em um quarto da área em estudo.

De maneira geral, todos os valores de RP foram baixos, variando de 1,12 a 1,45 MPa, considerado um dado importante e positivo, pois significa que o futuro plantio não sofrerá impedimento ao crescimento radicular, já que a faixa de variação dos valores de RP não representa valores críticos de 2 MPa (REICHERT et al., 2007) e nem é considerado restritivo de $3 \mathrm{MPa}$ (ZOU et al., 2000). A grande quantidade de resíduos depositados sobre o solo (cerca de $90 \mathrm{t}$ ) pode ter atenuado o processo de compactação do solo e seus efeitos, pois, segundo Braida et al. (2006), o material orgânico, nessa situação, dissipa parte da pressão exercida pelos rodados das máquinas.

Revista Árvore, Viçosa-MG, v.38, n.4, p.641-648, 2014

\section{CONCLUSÃO}

A umidade do solo influenciou a compactação, mas a colheita realizada em dia de chuva, três dias ou sete dias após a chuva, não influenciou, de forma diferenciada, a qualidade física do solo, pois a umidade nessas áreas foi estatisticamente igual.

As operações de colheita impactaram o solo até a profundidade de $10 \mathrm{~cm}$, causando compactação nas linhas de tráfego das máquinas, enquanto a eventual queda de árvores no momento do corte e o empilhamento de toras não provocaram alterações estruturais no solo.

A biomassa residual da colheita florestal foi importante para a minimização da compactação do solo causada pelo tráfego das máquinas.

A umidade do solo no momento das operações de colheita estava próxima da umidade da capacidade de campo, portanto a suscetibilidade do solo à compactação estava próxima da máxima compactação.

\section{REFERÊNCIAS}

BRAIDA, J. A. et al. Resíduos vegetais na superfície e carbono orgânico do solo e suas relações com a densidade máxima obtida no ensaio Proctor. Revista Brasileira de Ciência do Solo, v.30, n.4, p.605-614, 2006.

BAVER, L. D.; GARDNER, W. H. Física del suelos. México: Hispano-Americana, 1973.

BUSSCHER, W. J. Adjustament of flat-tipped penetrometer resistence data to a common water content. Transactions of the ASAE, v.33, n. 2, p.519-524, 1990.

CAMARGO, C. M. S. Compactação do solo causada pela colheita de Pinus taeda, pelo sistema de fuste (Tree-length). 1999. 110f. Dissertação (Mestrado em Engenharia Florestal) - Universidade Federal do Paraná, Curitiba, 1999.

CAVIGLIONE, J. H. et al. Cartas climáticas do Paraná. Londrina: IAPAR, 2000.

CULLEN, S. J.; MONTAGNE, C.; FERGUSON, H. Timber harvest trafficking and soil compaction in Western Montana. Soil Science Society American Journal, v.55, n.5, p.1416-1421, 1991. 
DIAS JÚNIOR, M. S. Avaliação quantitativa da sustentabilidade estrutural de um Latossolo vermelho amarelo situado em áreas experimentais da Cenibra: Relatório do projeto de pesquisa. Lavras: Universidade Federal de Lavras, 1999. 23p.

\section{EMPRESA BRASILEIRA DE PESQUISA}

AGROPECUÁRIA - EMBRAPA. Centro Nacional de Pesquisa do Solo. Manual de métodos de análise de solo. 2.ed. Rio de Janeiro: 1997. 212p.

FENNER, P. T. Compactação do solo. In: MACHADO, C. C. Colheita florestal. Viçosa, MG: Universidade Federal de Viçosa, 2002. p.375396.

HILLEL, D. Flow of water in unsaturated soil. In: HILLEL, D. Environmental soil physics. New York: Academic Press, 1998. p.203-241.

KLEIN, V. A. Física do solo. 2.ed. Passo Fundo: EDIUPF, 2012. 240p.

LOPES, E. S. et al. Avaliação da umidade na compactação do solo submetido ao tráfego de máquinas de colheita florestal. Revista Árvore, v.35, n.3, supl.1, p.659-667, 2011.

MARSILI, A. et al. Changes of some physical properties of a clay soil following passage of rubber and metal tracked tractors. Soil and Tillage Research, v.49, n.2, p.185-199, 1998.

MÓSENA, M.; DILLENBURG, L. R. Early growth of Brazilian pine (Araucaria angustifolia) in response to soil compaction and drought. Plant Soil, v.258, n.1, p.193-306, 2004.

OLIVEIRA JÚNIOR, E. D. Compactação do solo devido ao tráfego de carretas florestais com dois tipos de pneus inflados a duas pressões diferentes. 1998. 68f. Dissertação (Mestrado em Agronomia) Escola Superior de Agricultura Luiz de Queiroz, Piracicaba, 1998.

REICHERT, J. M.; REINERT, D. J.; BRAIDA, J. A. Qualidade dos solos e sustentabilidade de sistemas agrícolas. Ciência e Ambiente, v.27, p.29-48, 2003.

REICHERT, J. M.; SUZUKI, L, E. A. S.; REINERT, D. J. Compactação do solo em sistemas agropecuários e florestais: identificação, efeitos, limites críticos e mitigação. In: CERETTA, C. A.; SILVA, L. S.; REICHERT, J. M. Tópicos em ciência do solo. Viçosa, MG: Sociedade Brasileira de Ciência do Solo, 2007. p.49-134.

SAMPIETRO, J. A.; LOPES, E, S. Compactação de um Cambissolo e Neossolo submetidos a diferentes intensidades de tráfego de Feller Buncher e Skidder. Scientia Forestalis, v.39, n.90, p.265-272, 2011.

SEIXAS, F.; KOURY, C. G. G.; RODRIGUES, F. A. Determinação da área impactada pelo tráfego de forwarder com uso de GPS. Scientia Forestalis, v.68, p.178-187, 2003.

SEIXAS, F.; OLIVEIRA JÚNIOR, E. D. Compactação do solo devido ao tráfego de máquinas de colheita de madeira. Scientia Forestalis, v.60, p.73-87, 2001.

SEIXAS, F.; OLIVEIRA JÚNIOR, E. D.; SOUZA, C. R. Efeito da camada de resíduos florestais na compactação do solo causada pelo transporte primário da madeira. Scientia Forestalis, v.54, n.1, p.9-16, 1998.

SILVA, A. R.; DIAS JUNIOR, M. S.; LEITE, F. P. Camada de resíduos florestais e pressão de preconsolidação de dois Latossolos. Pesquisa Agropecuária Brasileira, v.42, n.1, p. 8993, 2007.

SILVA, R. S.; BARROS, F. N.; COSTA, L. M. Atributos físicos de dois Latossolos afetados pela compactação do solo. Revista Brasileira de Engenharia Agrícola e Ambiental, v.10, n.4, p.842-847, 2006.

SOJKA, R. E. Stomatal closure in oxygen-stressed plants. Soil Science Society of America Journal, v.154, n.1-2, p.269-280, 1992.

STEPNIEWSKI, W. et al. Effects of compaction on soil aeration properties. In: SOANE, B. D.; OUWERKERK, C. (Ed). Soil compaction in crop production. Amsterdam: Elsevier, 1994. p.167-189.

TORMENA, C. A.; SILVA, A. P.; LIBARDI, P. L. Caracterização do intervalo hídrico ótimo de um Latossolo Roxo sob plantio direto. Revista Brasileira de Ciência do Solo, v.22, n.2, p.573-581, 1998.

Revista Árvore, Viçosa-MG, v.38, n.4, p.641-648, 2014 
WARKOTSCH, W. The impact of harvesting operation on timber quality: causes and remedies. South African Forestry Journal, v.169, n.1, p.33-48, 1994.
ZOU, C. et al. Least limiting water range: A potential indicator of physical quality of forest soils. Australian Journal of Soil Research, v.38, n.5, p.947-958, 2000. 